\title{
Logic Mining for Financial Data
}

\author{
G. Felici ${ }^{1}$, M.A. Galante ${ }^{2}$, and L. Torosantucci ${ }^{3}$ \\ 1 Istituto di Analisi dei Sistemi ed Informatica del CNR, \\ Viale Manzoni 30 - 00185 Roma, Italy \\ felici@iasi.cnr.it \\ 2 Istituto di Analisi dei Sistemi ed Informatica del CNR, \\ Viale Manzoni 30 - 00185 Roma, Italy \\ galante@iasi.cnr.it \\ 3 Banca Nazionale del Lavoro \\ luca.torosantucci@bnlmail.com
}

\begin{abstract}
In this paper we consider a new strategy for supporting timing decisions in stock markets. The approach uses the logic data miner Lsquare, based on logic optimisation techniques. We adopt a novel concept of good session, based on the best return expected within a given time horizon. Such definition links indirectly the buying decision with the selling decision and make it possible to exploit particular features of stock time series. The method is translated into an investment strategy and then it is compared with the standard buy \& hold strategy.
\end{abstract}

\section{Introduction}

In this paper we investigate a new approach for the analysis and the forecasting of financial data. Such approach is based on the combination of Logic Data Mining with financial Technical Analysis. Logic Data Mining is a particular type of automated learning that derives from data a set logic rules that exploit the structure of the data, if present, and then allows to classify new records with a good level of accuracy. We use the Lsquare System [6], a logic data miner based on logic programming designed specifically for application to logic data that has some original features particularly useful in this context. Technical Analysis is based on a number of indicators that are computed from the values of a stock in different time intervals. It typically considers the opening, closing, minimum, and maximum values of a stock in one or more time intervals (e.g., the daily sessions) and computes from such values some indicators that suggest to buy or to sell the security under analysis. In this paper we briefly overview the basic concept of financial theory, provide a general overview of the method proposed, describe the learning algorithm used, and present computational results.

\section{Financial Theory and Learning Applications}

One of the most representative theories in finance is the Efficient Market Hypothesis $(\mathrm{EMH})$ of Fama ([4], [5]). This hypothesis states that security prices fully, instantaneously and rationally reflect all available information about that 
security. A price that fully and rationally reflects all available information implies that the market is perfectly rational and that every kind of information can be unequivocally interpreted. A price that instantaneously reflects all available information implies that the time reaction of the market is so fast that it is not possible to take advantage of new data. For these reasons, if the EMH is true, it is not possible to implement an investment strategy that constantly obtains greater returns than the market, usually represented by an index (e.g. the S\&P500). The discussion on market efficiency is not only academic. Generally, investors pick the asset of a portfolio by using either Fundamental Analysis or Technical Analysis (TA). The fundamentalist states that, in general, prices do not reflect the real value of the underlying firm. This implies that the market is not rational and the stock can be either overpriced or underpriced, according to the misunderstanding of the available information. The fundamentalist also believes that every mistake will be corrected by the market. On the contrary, the follower of Technical Analysis believes that the market, on average, is rational. If a market is really efficient, both technical and fundamental analysis can not constantly ensure greater returns than the market. For this reason, according to the EMH, the best strategy for an investor should be to buy and hold a representative portfolio of the market. After the first exhaustive claim of the EMH made by Fama in 1970, a great number of papers seemed to confirm unequivocally the efficiency of the market. At the same time, a lot of research showed the unpredictability of prices and markets. In the 1980s a few papers highlight the first anomalies regarding the efficient market hypothesis, but only at the end of that decade and the beginning of the 1990s the idea of the EMH was reconsidered. In 1989 Samuelson changed his original opinion [13] and stated that efficiency is not a perfect paradigm for the market. In his work, he recalls papers which show statistically significant deviations from the random walk and the fact that it is possible to find, on long time horizons, a tendency of the price. Several papers test the effectiveness of technical analysis by means of empirical research on specific timing strategy (among them, [3] [5], [11], [12]). The work presented in this paper falls into this last category, but is characterized by the application of Data Mining techniques, that provide a large number of non parametric, non linear and non statistic models to extract knowledge from observed data. Often, the complexity of such models requires sophisticated optimisation techniques. Such tools have appeared in financial literature in recent years and seem to play a key role to uncover the hidden and local regularities that may allow to beat the market in the short to mid term, contradicting the EMH in its strong form but not necessarily in its weak or semi-strong form. Their effectiveness is often to be accounted to their capability of using models that go beyond the standard linear models of multidimensional statistic regression, as shown in the many applications described in [8], [9], [10].

\section{An Overview of the Method}

In this section we describe the proposed method to determine the timing to buy and to sell a stock in a profitable way. We provide the list of the TA indicators 
used and the definition of good session; we consider the problem of identifying the right training period and the problem of when is it useful to learn from data. The learning tool Lsquare and its role in the whole picture will be treated in Section 4.

\subsection{The Learning Approach}

We intend to learn from a number of previous sessions whether the current session is good to sell or to buy a given stock. To accomplish this task we must start from the definition of a number of measures of each session and a way to identify whether a session is good or not. Such data, properly processed by a learning algorithm, may identify some relation between the measures of the session and its type (good, not good), that can be used for making a decision about the right operation to make in the current session. In the following we will refer to daily sessions (technically a session may be identified by any fixed time interval). We first provide some notation. We refer to $t$ as the current session (or day) where we want to make the decision. The Training Period TP is the number of days before $t$ that we want to analyse to support our decision. We indicate with $T$ the length in days of the training period. Each session $i \in T P$ is represented by a vector of $m$ measures $V_{i}=v_{i}^{1}, v_{i}^{2}, \ldots, v_{i}^{m}$. Each session can belong to one of two classes: class $A$ (buy) or class $B$ (don't buy). Then, we apply a learning algorithm to determine a classification rule into $A$ or $B$ of the sessions in the $T P$ based on their description by vectors $V_{i}, i \in T P$. Such rule is used to determine whether today's session, $S_{t}$, belongs to class $A$ or class $B$ and take appropriate action. In the next sections we will see how we determine the measures in $V_{i}$ and what determines the belonging of a session to $A$ or $B$.

\subsection{The Indicators of Technical Analysis}

Technical Analysis (TA) developed a great number of analysis instruments. Typically, four different types are considered: Trend Instruments, that identify the trend by means of straight lines which pass through as great a number as possible of local minimum and maximum of prices; Moving average, by which traders buy (sell) when the chart is over (under) its moving average; Oscillator Indicators, that are simple mathematical models which define critical situations of the market. The presence of a specific codified pattern (the codification is based on the analysis of the past) can describe the most probable consequence for the price in the future. Contrary to fundamental strategies, technical analysis does not use public information such as announcements of new prospects or balance sheet items; according to traders, the current price correctly reflects this information. Generally, four time series are used, which are composed of the highest price, the lowest price, the closing price and the open price of a significant period of time, called session. The length of the session depends on the time horizon of the single investor. The most common session is daily, but investors with a shorter time horizon can also use a shorter session, for example of an hour or five minutes. On the other hand, investors with a longer horizon can use a session 
of a week or a month. We have tested and implemented the most common TA indicators, on different time periods, and verified their effectiveness in spotting a good session when used as input data for the logic data miner. We omit for brevity a detailed description of the indicators listed below, as they are common knowledge in the financial literature: Moving Average (MA), Relative Strength Indicator (RSI), Stochastic Oscillator, P-Momentum, On balance volume (OBV), Price and Volume Trend (PVT), Volume Accumulation Oscillator (VAO).

The most serious obstacle to the investigation of technical analysis is his great number of instruments which can be combined to implement a timing investment strategy. Each instrument concentrates his analysis on a particular aspect of the market; even if a timing strategy based on one or more TA indicators may be reasonable from a financial point of view, it is unlikely to ensure a better result than the market in varying conditions. Moreover, each instrument generally depends on a set of parameters and these parameters depend on the current market and on the time horizon of the investor. Typically these parameters are thresholds which permit to establish if a stock is over- or under-bought. Unfortunately, there is not a rigorous method to fix them. We have tackled these two open problems in the following way. First, we have represented each session by a large number of indicators, with different values of $n$, and determined experimentally what set of indicators was providing better overall performances in our learning scheme. Once such reduced set was determined, we used it to represent each session, using the selected indicators to determine the components of the vectors $V_{i}$ for all sessions in the training period TP (see section 3.1). Second, we have avoided the choice of thresholds for the indicators using a particular feature of the learning method Lsquare that automatically maps rational values into intervals in an optimal way with respect to the learning task.

\subsection{The Good Sessions and the Timing Strategy}

One of the problems that is encountered when using a learning method for financial decision is what is to be considered a good (or bad) session; in other words, what are the classes (e.g., the $A$ and $B$ of section 3.1) that we would like to be able to recognize in the future to make good decisions. Such definition is strongly dependent on the investment strategy that will use this information. In this paper we have adopted an original definition of good session that is related with the time horizon in which the trader intends to operate. Let us assume that this time horizon, indicated with $h$, is a measure of the frequency in which we want to operate on the stock (for example, 10 days). For a given session $i$, let close $_{i}$, open ${ }_{i}, \min _{i}, \max _{i}$, be the opening, closing, minimum, and maximum prices in the session, respectively. For session $i$, let the Best Return Within $h$ be defined as:

$$
B R W_{h}(i)=\max _{j=i, i+h} \frac{\text { close }_{j}-\text { close }_{i}}{\text { close }_{i}} .
$$

Such value represents the maximum increase in value that could be obtained on that stock buying it on the closing price of day $i$ and selling it within the 
next $h$ days, at closing price. For a given training period $T P$, consider now the distribution of $B R W_{h}(i), i \in T P$, and take the value $\lambda$ as the splitting point that separates the highest $25 \%$ of that distribution from the rest. Now, assume one can correctly predict whether today's session $t$ has $B R W_{h}(t)$ higher than $\lambda$ or not. This would imply a very simple investment strategy that guarantees returns that are significantly higher than the market. Obviously such strategy cannot be applied in practice, as we cannot know in advance the value of $B R W_{h}(t)$; but, if we can estimate with a good level of accuracy whether it is above the current $\lambda$, we can expect high returns and sell the stock within $h$ sessions as soon as it rises above $\lambda$. On the other hand, if the prediction turns out to be incorrect, that is, if the stock price does not rise as much as $\lambda$ in $h$ sessions, we sell the stock and tolerate that potential loss.

\subsection{The Identification of the Training Period}

The strategy described above is applied anew in each session $t$, computing new indicators and new classification rules from a $T P$ of given length determined with a sliding time window. A relevant question to answer is what is the appropriate length of the training period; the length is obviously dependent from the time horizon $h$. Such parameter requires proper tuning and has been found that a value of 75 days when $h=10$ provides good performances for the stocks analysed. Another crucial issue is whether the information contained in the training period is intersting for learning or not. In this setting, two major considerations arise. As far as the current trend of the stock is concerned, an immediate information is found in the value of $\lambda$. If in session $t$ its training period $T P$ has $\lambda<0$, we know that even in the best $25 \%$ of the sessions in TP there are sessions that do not provide any gain in the value of the stock. In such case the session is skipped, no learning is applied and the stock is not purchased. When the distribution of $B R W_{h}(i), i \in T P$, is very flat, the information available in $T P$ is not sufficiently varied to provide good support for differentiation of the two classes. It may also happen that all sessions in the training period are positive sessions: they provide a value of $B R W_{h}(i)$ sufficiently large, and thus the recognition problem is not relevant for the success of the investment. To apply this consideration is a systematic way, we adopt a filtering rule based on the mean $\mu$ and the variance $\sigma$ of the distribution of $B R W_{h}(i), i \in T P$ and set a threshold $\sigma^{o}$ and two thresholds $\mu_{\text {low }}$ and $\mu_{\text {high }}$ to implement the above considerations.

\section{The Learning System Lsquare}

The learning tool used in this application, Lsquare, is a particular learning method that operates on data represented by logic variables and produces rules in propositional logic that classify the records in one of two classes. The general scheme of this method is the one of automatic learning, where the records presented in a training set are used to infer the rules that link the class variable with some measures; these rules are then used to classify new records and predict their class. Here we intend to apply this method in each daily session, using as 
training data the previous $T$ sessions, represented by a vector $V_{i}$ of measures derived from a set of TA indicators. The training set, or TP, is divided into class $A$ (good sessions, that have the value of the Best Return Within $h$ days larger than $\lambda$ (see Section 3.3)), and class $B$ (the other sessions in TP). The choice of Lsquare is motivated by the fact that it uses a logic representation of the description variables, that are to all extents logic variables, and of the classification rules, that are logic formulas in Disjunctive Normal Form (DNF). Such feature enables to analyse and interpret the classification formula also from the TA point of view, exploiting the relation between the indicators and their threshold values. The learning of propositional formulas from data is tackled also by other learning methods, such as the strongly heuristic, simple and widely used decision trees (originally proposed in [2]), to the more sophisticated LAD system ([1]), to the greedy approach proposed in [14]. The Lsquare system and some of its additional components have been described in [6], and its detailed description is out of the scope of this paper. Here we simply mention the fact that the rules are determined using a particular problem formulation that amount to be a well know and hard combinatorial optimisation problem, the minimum cost satisfiability problem, or MINSAT, that is solved using a sophisticated program based on decomposition and learning techniques [15]. The DNF formulas identified are composed of conjunctive clauses that are searched for in order of coverage of the training set, and are formed by few clauses with large coverage (the interpretation of the trends present in the data) and several clauses with smaller coverage (the interpretation of the outliers in the training set). Lsquare is equipped with an automatic method to map continuous variables into binary ones (a complete description of this method may be found in [15]). This approach tries to solve the problem related with the choice of the thresholds in the use of TA indicators: we can in fact use the natural representation of many of them (e.g., the 0-100 value of the indicator RSI see section 3.2) and let the system choose the thresholds at which these indicators become useful to differentiate the good from the bad sessions.

\section{Experiments}

In this section we describe the results obtained by the application of our method to time series of stocks. In each experiment we consider an experiment period, in which each day represent a separated session. We assume to dispose of a fixed amount of capital to invest, say $K$, available at the beginning of the experiment period. Some necessary hypotheses were also made, that may reduce part of the risks related with the application of investment strategies in real markets: we do not allow to buy or sell a portion of the available capital, we assume to successfully buy or sell the stock at the closing price of each session, and we discount a fixed $0.02 \%$ transaction cost each time the stock is bought. Having chosen a training period $T P$ of length $T$, a time horizon $h, \sigma^{o}, m u_{l o w}$, and $m u_{h i g h}$, we then perform the following steps (where $t$ is the current session and $T P$ is the set of sessions from $t-T-1-h$ to $t-1-h)$ : 
INVESTMENT_STRATEGY $\left(T P, h, \sigma^{o}, m u_{l o w}, m u_{h i g h}\right)$

1. Initialize. Set $i=0, d^{*}=-1, \lambda^{*}=-1$, goto step 1

2. Classify $T P$. For each session $i \in T P$, compute $B R W_{h}(i)$. From the distribution of $B R W_{h}(i), I \in T P$, compute $\lambda, \mu$ and $\sigma$. For each session $i \in T P$, if $B R W_{h}(i)>\lambda$ define session $I$ to be in set A, and to be in set B otherwise; goto step 2

3. Evaluate bad. If $(\lambda \leq 0)$ or $\left(\sigma<\right.$ sigma $^{o}$ and $\left.\mu<m u_{l o w}\right)$ then go to step 6 , else goto step 3

4. Evaluate good. If $(\lambda>0)$ and $\left(\sigma<s i g m a^{o}\right.$ and $\left.\mu>m u_{h i g h}\right)$ then go to step 5, else goto step 6

5. Learn and Classify. Process the sessions in $T P$ by Lsquare to learn classification rules for $A$ and $B$. Apply such rules to current session $t$. If session $t$ is classified in $A$, goto step 5 , else goto step 6

6. Buy. If capital is available, use all of it to buy stock. set $\lambda^{*}=\lambda$ and $d^{*}=t+h$ and goto step 1

7. Sell. If $B R W_{h}(t)>\lambda^{*}$ or $t>=d a y^{*}$, sell all stock and goto step 1

We have considered two different stocks in different periods, making sure that the data obtained was correct and complete. The main parameters involved were initially tuned with several runs, and then used in the two experiments described below. We use a value of 10 for the time horizon $h$ and 75 days as training period. The decision thresholds $\sigma^{o}, m u_{l o w}$, and $\mu_{h i g h}$ were set to 1 , 2.0 and 3.0 respectively. The first experiment refers to the daily sessions of the Future Bund (the future on the decennial german government bond), in the 200 days from November $22^{\text {nd }} 2002$ to October $10^{t h}$, 2003. In this period the Bund has a positive trend with little fluctuations and our system is able to improve on Buy \& Hold strategy by a significant ratio (see Figure 1, a)). At the end of the period, the Buy \& Hold reports a gain of $1.049 \%$, outoperformed by the $5.003 \%$ obtained by the proposed method. In the second experiment we take into account a stock with more fluctuations, in order to test the capability of the method in a different situtation. The consider case is the Alitalia bond from August $27^{\text {th }} 2004$ to June $6^{t h}, 2005$. While Buy \& Hold registers a loss of $-4.639 \%$, the proposed

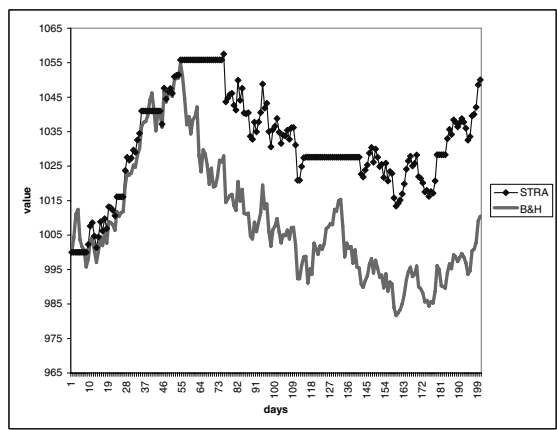

(a)

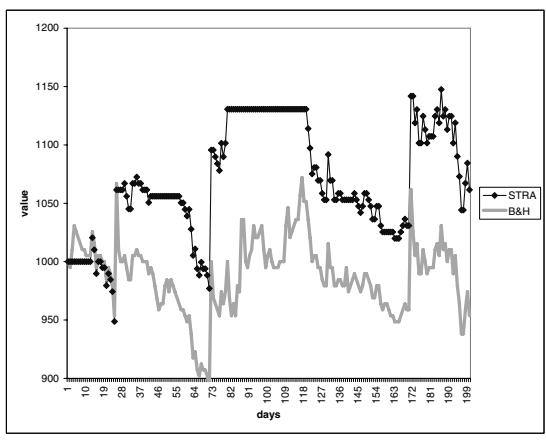

(b)

Fig. 1. Comparison with Buy and Hold for German Bund (a) and Alitalia (b) 
method obtains, at the end of the period, a positive $6.148 \%$ (see Figure 1, b)). We also note that the proposed strategy shows better performances for each session of the experiment period.

\section{References}

1. Boros E., Ibaraki T., Makino K., 1999, Logical analysis of binary data with missing bits Artificial Intelligence 107, 219-263.

2. Breiman, Friedman, Olshen, Stone, 1984, Classification $\&$ Regression Trees,Pacific Grove, Wadsworth.

3. Elton-Gruber, 1995, Modern Portfolio Theory and Investment Analysis, John \& Wiley \& Sons Inc.

4. Fama, E. Efficient capital markets: A review of theory and empirical work, "Journal of Finance 25, 383-417, 1970

5. Fama "Efficient capital markets: II", Journal of Finance 46, 1575-1617, Dic. 1991

6. Felici G., Truemper K., 2001, "A MINSAT Approach for Learning in Logic Domains", INFORMS Journal on Computing, Vol.13, No.3, pp. 1-17

7. Frankfurter G., McGoun E., Anomalies in finance: what are they and what are they good for? International Review of Financial Analysis 10 (4) (2001) 407-429.

8. Leigh W., Modani N., Purvis R., Roberts T., Stock market trading rule discovery using technical charting heuristics, Expert Systems with Applicatyions, 23 (2002), $155-159$

9. Leigh W., Purvis R., Ragusa J.M., Forecasting the NYSE composite index with technical analysis, pattern recognizer, neural network, and genetic algorithm: a case study in romantic decision support, Decision Support Systems 32 (2002), 361-377

10. Kovalerchuk B., Vityaev E., Data Mining in Finance: Advances in Relational and Hybrid Methods, Kluwer Academic Publishers, Norwell Massachussetts, 2000

11. Pinches G.E., The Random Walk Hypothesis and Technical Analysis, Financial Analysts Journal, March-April 1970.

12. Pring M.J., Technical Analysis Explained : The Successful Investor's Guide to Spotting Investment Trends and Turning Points, McGraw-Hill; 4 edition (February 20, 2002)

13. Samuelson, The Judgement of Economic Science on Rational Portfolio Management: Indexing, Timing, and Long Horizon Effect, Journal of Portfolio Management, n 1, 1989.

14. Triantaphyllou, E., and A.L. Soyster, On the Minimum Number of Logical Clauses Which Can be Inferred From Examples," Computers and Operations Research, Vol. 23, No. 8,1996, pp. 783-79 9

15. Truemper K. Design of Logic-Based Intelligent Systems, Wiley-Interscience, 2004 